Les Cahiers du Gres

\section{Pratiques d'accueil et traitement des discriminations}

Les permanences d'une association antiraciste en France

\section{Mireille Eberhard}

Volume 4, numéro 1, printemps 2004

URI : https://id.erudit.org/iderudit/009716ar

DOI : https://doi.org/10.7202/009716ar

Aller au sommaire du numéro

Éditeur(s)

Groupe de Recherche Ethnicité et Société

CEETUM

ISSN

1499-0431 (imprimé)

1499-044X (numérique)

Découvrir la revue

Citer cette note

Eberhard, M. (2004). Pratiques d'accueil et traitement des discriminations : les

permanences d'une association antiraciste en France. Les Cahiers du Gres, 4(1),

75-87. https://doi.org/10.7202/009716ar
Les Cahiers du Gres Série Diversité urbaine Groupe de Recherche Ethnicte et Sodete d'utilisation que vous pouvez consulter en ligne.

https://apropos.erudit.org/fr/usagers/politique-dutilisation/ 


\section{Notes de recherche}

\section{Pratiques d'accueil et traitement des DisCRIMInNATIONS. Les PERMIANENCES D'UNE ASSOCIATION ANTIRACISTE EN FranCE}

\section{Mireille Eberhard}

T a lutte contre les discrimina-

Utions racistes est très récente en France : la prégnance et le poids d'un idéal républicain ont longtemps rendu difficile l'émergence de cette question, lui préférant une approche en terme d'intégration. L'approche républicaine, qui ne considère que des individus, nie toute distinction, individuelle ou collective, fondée sur "l'origine ou la race ». Or la discrimination renvoie justement à l'application d'un traitement à la fois différent et inégal à un groupe ou à une collectivité ${ }^{1}$ en fonction de traits, réels ou imaginaires, socialement construits comme " marques négatives » ou « stigmates » (De Rudder, Poiret, Vourc'h, 2000). Le contexte républicain délégitimant, voire interdisant la caractérisation même de ces groupes discriminés, l'existence de traitements différenciés a longtemps été occultée dans l'espace public français. L'insuffisance et le caractère inopérant des principes formels d'égalité de droits et d'égalité de traitements ont conduit à la remise en cause de l'approche exclusive en termes d'intégration. L'obligation faite aux pays européens d'appliquer l'article 13 du traité d'Amsterdam (article qui intègre la lutte contre les discriminations dans les grandes missions de l'union) a aussi pesé en faveur de ce changement de perspectives. Finalement, la prise en compte de la question des discriminations par les pouvoirs publics peut être datée du 21 octobre 1998, date à laquelle la ministre de l'emploi et de la solidarité, lors d'une communication au conseil des ministres, déclare faire de la lutte contre les discriminations l'une des priorités de sa politique. S'en est suivie la création et la mise en place d'un dispositif public de lutte contre les discriminations, ainsi que le renforcement $d u$ dispositif législatif existant, notamment avec l'adoption en 2001 d'une loi « relative à la lutte contre les discriminations $).$

C'est dans ce nouveau contexte politique et institutionnel que l'association MRAP (Mouvement contre le racisme et pour l'amitié entre les peuples) a lancé, fin 2000 , une campagne nationale de lutte contre les discriminations intitulée "discrimination, ouvrons les yeux ", se saisissant de ce fait d'une problématique encore peu soulevée par le milieu associatif 
antiraciste français. L'action antiraciste portait, jusque-là, plus sur le racisme appréhendé en termes idéologiques, voire politiques, et ciblait finalement plus le droit de vote des étrangers ou les idées du Front National que les pratiques racistes en tant que telles. Le passage à une action en termes de " lutte contre les discriminations » marque donc, pour l'association, une manière différente de se saisir de la question du racisme et de ses modalités d'inscription dans la réalité. Coordonnée par les instances nationales du MRAP, cette campagne s'appuie sur un réseau de 49 comités locaux au sein desquels a été mise en place la partie la plus importante, mais également la plus novatrice de cette campagne, celle portant sur la création de structures d'écoute et d'assistance aux victimes de discriminations. Ces permanences d'accueil, tenues par des militants ${ }^{2}$, ont pour vocation l'écoute, le soutien et le suivi juridique des victimes et des témoins de discriminations racistes. C'est à travers leur analyse que je m'intéresserai à la manière dont une association antiraciste telle que le MRAP se saisit et traite la question des discriminations racistes.

Par son objet, mais également par le type d'engagement que constitue la tenue de permanences - tant en termes de savoirfaire que de manières de faire cette campagne constitue une nouvelle forme d'engagement et de pratiques pour le MRAP et ses militants. L'accueil représente, en effet, une activité militante qui ne se situe plus sur le terrain de la contestation, mais sur celui de l'action. La « cause " pour laquelle la personne milite est incarnée par une situation concrète, par une personne qui fait la démarche de se rendre à une permanence sur les discriminations pour y exposer sa situation. Le racisme n'est alors plus appréhendé par le militant en tant qu'idéologie, mais comme une réalité vécue, quotidienne, matérielle. Pour ce dernier, les compétences et les qualifications requises pour tenir une permanence sont multiples, et il doit disposer d'un minimum de connaissances, tant sur le fonctionnement du dispositif public de lutte contre les discriminations, sur la législation contre le racisme et les discriminations, que sur l'attitude à adopter face à une personne venue exprimer une souffrance. Sans aller jusqu'à une professionnalisation à proprement parler, l'accueil lui demande donc une spécialisation, un savoir-faire nouveau qu'il doit s'approprier. Ainsi, l'accueillant doit être en mesure d'apporter à la victime une écoute, mais également des réponses et des modes de traitement de son cas pouvant se situer sur un terrain non contentieux (rappel à la loi, demande d'explication à l'auteur présumé de la discrimination, renvoi vers d'autres structures...), aussi bien que sur un terrain contentieux (aide à la rédaction de plainte, accompagnement au tribunal, renvoi vers un avocat, constitution de partie civile du MRAP...). La finalité de ces permanences d'accueil est affichée comme multiple par 
l'association qui les présente comme ayant une vocation " d'écoute, de soutien et du suivi des victimes ou témoins de discriminations racistes $॥$. Il ressort de cette définition une oscillation entre un pan « écoute » et un pan « juridique » de l'accueil. Nous verrons que cette oscillation dans la modalité de traitement des discriminations racistes peut être expliquée par la difficulté à appréhender, à objectiver la " substance ॥ même de cette réalité sociale pour les acteurs sociaux.

Ici, je confronterai les finalités « affichées » de ces permanences, leur matérialité, et leur effectivité. J'aborderai donc les modalités de traitement des discriminations mises en œuvre par une association, mais également la difficulté devant laquelle se trouvent les militants pour influencer cette réalité, du fait de l'ambiguïté de cet objet social et de la multiplicité de ses inscriptions dans le réel qui la rend difficile à objectiver. C'est à la réalité de l'accueil que je m'intéresserai, m'appuyant sur les pratiques et les discours des deux types de protagonistes que ces permanences mettent en jeu : les plaignants et les militants. Les résultats exposés ici proviennent d'une enquête de terrain effectuée au MRAP, dans le cadre de mon doctorat $^{3}$. Ils reposent 1 ) sur des informations que j'ai recueillies, par entretiens ou discussions plus informelles, auprès de militants en charge de l'accueil dans huit comités locaux ${ }^{4}$ engagés dans la campagne "Discriminations, ouvrons les yeux »;2) sur des ob- servations que j'ai effectuées au sein des permanences d'accueil du comité de Paris. Plus spécifiquement, je me suis appuyée ici sur l'analyse de 25 entretiens d'accueil victime-militant. Mon but ici n'est pas de construire une typologie de ces victimes ou de ces militants, mais de cerner le type de connaissance qu'apporte l'analyse de leurs interactions en termes de compréhension et de définition de la notion de discrimination raciste ${ }^{5}$.

\section{Le poids de l'approche judi- ciaire}

La lutte contre les discriminations est fréquemment mise en avant comme une lutte se situant sur le terrain du droit, voire comme un "combat de droit", selon l'expression de Didier Fassin. L'inscription du MRAP dans cette lutte ne diffère pas de cette approche, le droit étant omniprésent dans la publicité qui est faite de ces permanences auprès du public : au recto d'une carte postale présentant la campagne figurent, par exemple, deux extraits du code pénal ayant trait aux discriminations. Cette prépondérance du droit se voit également dans le type de sessions de formation que la coordination nationale a organisées pour les militants des comités locaux dans le but de les aider à mener au mieux les permanences d'accueil : sur les 26 formations qui ont été dispensées en 2001 et 2002,18 sessions ont eu pour sujet « la législation contre le racisme et les discriminations $॥$, alors que seule- 
ment huit ont abordé « l'écoute de victimes et témoins de discrimination $॥$.

Au niveau de la pratique militante, cette approche judiciaire de la discrimination permet aux militants de traiter de façon concrète, matérielle, la situation de la personne qu'ils ont en face d'eux. Elle répond ainsi à une certaine " utilité sociale » du militantisme, militer renvoyant à la volonté de lutter, mais aussi d'agir, pour ou contre une cause. La volonté d'avoir un impact, une prise directe sur la réalité est donc l'une des finalités des militants, et le droit peut alors être présenté comme un outil dont ils peuvent se saisir pour y parvenir : "Pour moi, le droit c'est un moyen de militer et de faire en sorte de faire avancer un certain nombre de dossiers, et de lever un certain nombre d'injustices ». Pour les victimes, l'aspect symbolique de la sanction représente une attente très forte, puisqu'elle s'apparente à une reconnaissance publique de l'illégitimité du traitement qu'elles ont subi. D'ailleurs, rares sont les personnes qui vont en justice pour une réparation matérielle à proprement parler (de type dommages et intérêts ou réintégration d'un poste au sein d'une entreprise par exemple). Pour une grande part, l'attente des plaignants par rapport à ce type de démarche se situe dans le champ de la reconnaissance, de la réhabilitation personnelle: «Pour nous, c'est pas l'argent qui nous intéresse. On veut une réparation du préjudice moral. On veut être rétabli dans notre dignité et pour cela, il n'y a que le juge qui puisse le dire " il y a des choses qu'on peut faire et des choses qu'on ne peut pas faire. " "Qu'ils nous respectent! C'est tout ce que je demande moi. Je demande ni argent, ni rien. Mais ces gens, il faut les condamner! C'est pas juste. " La réponse judiciaire est également, pour la victime, une manière de se convaincre de sa non-responsabilité dans ce qui lui arrive : « Psychologiquement, j'en ai besoin; de savoir qu'elles soient reconnues coupables, parce que pendant pas moins d'un an je pensais être la coupable. Je pensais que c'était moi qui avais commis une erreur. ॥

Dans ce processus de réhabilitation de son histoire, de sa dignité, l'épisode judiciaire est fréquemment mis en avant par la victime comme une étape décisive permettant de "passer à autre chose ॥: "Je veux que justice soit faite! Pour moi, la question sera réglée quand la justice aura fait son travail, qu'ils auront été condamnés. Je serai libérée, moralement ». "Je veux pouvoir tourner la page ॥. « Dans un premier temps, on attendait un soutien parce qu'on a été très ébranlé par cette situation. Dans un deuxième temps, on souhaitait une reconnaissance de la faute pour nous aider à comprendre et à digérer ce qui s'était passé. Et puis dans un dernier temps, un changement pour l'avenir ». Ce dernier extrait montre également que le fait de porter son affaire devant la justice ne relève pas uniquement d'un acte " individuel » pour la personne, mais répond également à la volonté de faire en 
sorte que ce qu'elle a vécu ne puisse se reproduire. L'accent n'est alors plus mis sur le préjudice personnel en lui-même, mais sur l'exemplarité et l'impact de sa dénonciation : "J'ai décidé de me battre. Pour moi, mais aussi pour que ça n'arrive pas aux autres: pour que ça ne se reproduise plus dans cet établissement ni dans ce département ». «'ai voulu aller jusqu'au bout de cette démarche pour moi et pour les autres, ceux qui $n$ 'ont pas les moyens, ceux qui n'ont pour vivre que le boulot dont on les prive abusivement ". "J'ai été insulté, je ne veux pas que ça se reproduise. Je ne veux pas que mon petit frère vive une situation similaire $॥$.

Pour les victimes qui souhaitent porter leur affaire devant les tribunaux, l'association constitue un lieu de ressources juridiques. Non seulement les accueillants ont un minimum de connaissances juridiques, mais l'association dispose d'un service juridique spécialisé dans ce type de contentieux et sur lequel les militants peuvent s'appuyer dans leurs démarches d'aide et de soutien juridique aux victimes. Le MRAP est également en mesure d'orienter les personnes vers des avocats avec lesquels il travaille, voire d'accompagner la victime dans sa plainte, en se constituant partie civile à ses côtés, lorsque son dossier est suffisamment étayé. C'est pourtant à ce dernier niveau, celui de l'établissement des faits, que le bât blesse. Et, bien qu'il existe peu d'écrits scientifiques sur la question des discriminations, tous s'ac- cordent à déplorer les limites de l'approche judiciaire concernant l'extrême difficulté qu'il y a en droit français à apporter la preuve non seulement de l'acte discriminatoire, mais également de l'intentionnalité. Finalement, le traitement judiciaire des discriminations reste, dans les faits, marginal, ne concernant qu'un très faible nombre de cas $^{6}$. La portée pragmatique des textes législatifs est donc très faible, voire nulle. Et tout se passe comme si le juridique imposait un poids, une importance dans l'orientation de la lutte contre les discriminations qu'il est, au final, incapable d'assumer dans la pratique.

La formation juridique donnée au militant apparaît alors comme abstraite, idéelle : la législation leur est expliquée très en détails, tout comme les différents contentieux pouvant être qualifiés de discrimination, le type de tribunaux qu'ils peuvent saisir, le type de démarches qu'ils peuvent mettre en œuvre lorsqu'ils rencontrent tel ou tel cas « d'école »... Or toutes ces dispositions s'avèrent peu en phase avec la réalité qu'ils ont à traiter au sein des permanences. La prépondérance de l'approche judiciaire dans l'orientation de ces permanences finit donc par laisser perplexe. Face aux limites de l'action proprement judiciaire, les militants peuvent être d'autant plus déconcertés car c'est à eux qu'il incombe de gérer la déception des victimes: "Les victimes nous trouvent trop exigeants sur la façon dont il faut rapporter la preuve. Mais la rigueur de la 
preuve nous permet également de ne pas accuser sans fondement non plus ». «Beaucoup de gens peuvent être déçus par ce qu'on leur a dit. Ils ne sont pas forcément contents de la réponse. Le MRAP n'est pas forcément en mesure de répondre à une telle demande ». Les militants doivent également faire avec leur propre frustration, engendrée par leur impuissance face à un fait social dont ils saisissent toute la réalité (symbolique comme matérielle) mais qu'ils sont incapables d'objectiver: "Des fois, on sent qu'il y a du racisme. Le problème c'est que pour obtenir une réponse juridique, il faut une qualification ». « La discrimination est une notion difficile à cerner. [...] Quand les gens nous expliquent leur cas, difficile de prouver que quelqu'un a été refoulé par rapport à la discrimination. On dira qu'il a pas le profil si c'est dans l'emploi, pour le logement on dira que c'est un problème de paye $"$.

\section{Une notion ambiguë, difficile à objectiver}

Les limites de l'approche juridique ne se cantonnent pas uniquement aux difficultés liées à la preuve, mais également à la conception limitée du racisme et des discriminations qu'elle sous-tend. L'approche juridique impose en effet un schéma binaire - une victime / un coupable, tous deux clairement identifiés - dont elle a du mal à se départir. Non seulement ce schéma s'avère manichéen mais, de surcroît, il individualise et décontextualise la discrimination. Cette dernière n'est alors pas ap- préhendée en tant que processus s'inscrivant dans des rapports sociaux, mais comme un résultat. Finalement, comme le souligne Fassin, « la judiciarisation de l'inégalité illégitime induit un processus d'essentialisation de la discrimination » (Fassin, 2002). Or on ne peut considérer les caractéristiques ethniques ou phénotypiques d'un individu comme une variable indépendante qui induirait à elle seule tel ou tel comportement: elles s'insèrent dans des rapports sociaux qui les mettent plus ou moins en saillance et qui les instrumentalisent. La plupart du temps, les contextes dans lesquels la discrimination est produite sont intrinsèquement concurrentiels et inégalitaires, que l'on pense à la recherche d'un logement ou au milieu du travail. La variable ethnique ou phénotypique vient alors exacerber le rapport de forces inégalitaires, mais elle ne le crée pas de toute pièce.

Pour illustrer ces propos, je m'appuierai sur l'expérience d'un plaignant qui, à maintes reprises, s'est vu refuser l'accès à un poste de maître de conférence : « Le problème c'est que déjà, parce que moi j'ai des amis Français, des Dupont, qui ont le même problème. Ce que je suis en train de dire, c'est que déjà des Philippe, ils ont des problèmes, alors un Nordine... Mais l'une des difficultés, c'est que la sélection n'est pas anonyme [...] J'aimerais que quelqu'un qui fait une thèse fasse des statistiques ou une sorte de recherche sur le nombre de diplômés d'origine, c'est-àdire des Français d'origine 
multicouleur, et voir le pourcentage de ces gens là qui ont un poste qui leur correspond ou qui ont juste un poste, même si ça ne leur correspond pas. J'aimerais bien voir les statistiques ". L'intérêt de cet exemple réside dans le fait que la discrimination dont s'estime victime cette personne vient se greffer dans un monde de concurrence exacerbée, l'ouverture au concours d'un tel poste suscitant un grand nombre de candidatures. En principe, la sélection des candidats s'appuie sur des critères « objectifs » de leur dossier, selon des éléments méritocratiques, perçus comme légitimes. Cependant, le fait que cette sélection ne soit pas anonyme rend possible un mode de sélection certes illégitime, mais complètement informel donc inattaquable. Dans cet exemple, il semble que le plaignant ait conscience du fait qu'individuellement, il lui est impossible de prouver une quelconque discrimination, la moindre qualification ou la moindre adéquation au poste pouvant toujours être invoquée pour argumenter son éviction. Au final, il justifie la discrimination qu'il subit individuellement en invoquant une discrimination indirecte et systémique, concrétisée par une disqualification répandue et collectivement partagée.

La notion de discrimination indirecte est toutefois peu comprise par les militants, dont la perception se cantonne le plus souvent au schéma dominant d'appréhension de la discrimination où responsables et victimes sont identifiables.
La discrimination indirecte peut alors en amener certain à remettre en cause leurs propres pratiques, ou du moins à les interroger. Ce fut le cas pour un militant qui s'aperçut que la discrimination existait dans son entreprise sans qu'il s'en aperçoive et sans qu'elle soit intentionnelle : il se rendit compte que le fait d'embaucher uniquement des enfants du personnel, lorsque ce personnel est lui-même composé uniquement de personnes d'origine française, contribuait indirectement à écarter toutes personnes d'origine étrangère, ce qui constituait une forme de discrimination à laquelle il «n'avait jamais songé ». Cette anecdote met en lumière le changement de perspective et la remise en cause des schèmes de compréhension et de perception du racisme que peut être susceptible d'induire l'introduction de la problématique discriminatoire chez les militants anti-racistes. Outre la difficulté qu'ils peuvent avoir à la penser, ces derniers se heurtent également à l'ambiguïté même de l'objet qu'ils ont à traiter, du fait de la diversité et de la complexité des modalités d'inscription du racisme et de l'ethnisme dans la réalité des personnes qui le subissent.

Les personnes qui se rendent aux permanences d'accueil dénoncent fréquemment des pratiques discriminatoires pouvant être assimilées à du harcèlement, qui prennent la forme de brimades quotidiennes : «C'est une espèce de harcèlement haineux, le moindre prétexte. Je demande 
deux jours de congé qu'il me reste, on me dit "l ah non! mais monsieur mais vous avez une réunion vous ne pouvez pas ». C'est de la mauvaise foi, c'est de la saloperie quoi, il n'y a pas d'autres mots. [...] Elle cherche la querelle. Du style : elle arrive après moi le matin, je suis au téléphone avec un directeur d'école, elle se pointe devant ma porte avant la réunion et dit "si vous arriviez à l'heure vous ne passeriez pas vos coups de fil personnels ॥. Ici, la discrimination s'apparente à du racisme "voilé », et ses modalités d'inscription dans la réalité sont d'autant plus pernicieuses que le "fait raciste » (ou ethniste) proprement dit n'est pas manifeste. Encore une fois, l'approche dominante trouve ses limites, du fait de l'impossibilité de prouver que le traitement subi est directement lié à l'origine, réelle ou supposée, de la personne.

L'ambivalence de cette notion de discrimination résulte également de sa polysémie et de sa volatilité. Le terme, de par son suffixe "ion », désigne à la fois un comportement et le résultat qui en découle. Or, comme le souligne Saïd Bouamama, tout comportement discriminatoire ne débouche pas inéluctablement sur une situation discriminatoire (Bouamama et Benyachi, 2000). Je citerai l'exemple de cette personne qui s'est vue demander, au cours d'un entretien d'embauche, si elle aimait Zidane et si elle mangeait du couscous. Ce comportement peut être qualifié de discriminatoire, puisque le traitement dont cette personne fait l'objet résulte d'une imputation, d'une réduction de son identité à ses origines (réelles ou supposées) et à son phénotype. Au final, elle peut très bien finir par être embauchée. Cependant, on ne peut sous-estimer la violence symbolique que représente pour les plaignants cette assignation, ce renvoi ou du moins cette référence implicite, permanente et diffuse, à leur couleur ou à leur origine. C'est souvent la prégnance de ce que Philomena Essed nomme l'uevery day racism» (Essed, 1991) qui pousse les plaignants à se rendre aux permanences du MRAP, malgré la narration, au départ, d'un fait précis. Pour utiliser une image, je dirais que si c'est une goutte d'eau qui les fait se déplacer, c'est avant tout parce que le vase est plein. Le traitement de la discrimination raciste ne peut donc pas s'en tenir aux seuls aspects «matériels» que constituent l'entrave dans l'accès à un logement, à un déroulement de carrière ou encore à une boîte de nuit. Car si les discriminations jouent un rôle privatif en termes de droit, elles jouent également un rôle privatif en termes de reconnaissance, de respectabilité, de dignité, voire d'accès à un statut.

\section{Écoute et souffrance}

Les répercussions induites par les discriminations dans le vécu des personnes vont souvent bien au-delà de l'acte discriminatoire lui-même. Elles ne se limitent pas à la seule victime, mais touchent également son entourage familial, ce qui est régulièrement mis en avant par les victimes lors des entretiens d'accueil : « Aujourd'hui, ça 
nous fait mal d'avoir été obligés de baisser la tête et de partir, c'est vrai que c'est pas facile à assumer. Et le fait de ne pas pouvoir montrer à mes enfants qu'en tant que maman je n'ai pas pu faire tout ce qui était en mon pouvoir pour les aider, c'est très dur ». "Je suis marié, j'ai pas d'enfant. Heureusement. Parce que sinon je ne sais pas ce que je vais faire! mais c'est vrai que... Heureusement ma femme je l'ai connue il y a 10-12 ans. Heureusement on se connaît depuis longtemps, mais malgré ça, il y a des problèmes. On a beaucoup des problèmes. Et moi je ne me rends pas compte, mais elle me dit que j'ai changé, que je m'énerve pour un oui ou pour un non ". "Je reçois du courrier à la maison c'est des recommandés sans arrêt. Ma femme a même une procuration permanente pour pouvoir prendre mes lettres. Mes gosses, c'est vrai qu'ils vont pas bien. J'ai un fils qui s'est mis, à la première histoire, à devenir obèse : il n'est pas bien. $C$ 'est terrifiant. Il $y$ en a un qui dit " si papa perd son travail, qu'est ce qu'on va devenir ? ॥ c'est une inquiétude de tout le temps. De temps en temps il m'appelle au bureau, il me dit : papa il y a le facteur qui est passé, t'inquiète pas, il n'y a pas de lettre recommandée hein! »).

Les discriminations induisent aussi une remise en question des systèmes d'idées, de représentations du monde, voire de croyances et de valeurs de la victime, du fait de la restriction du champ des possibles qu'elles engendrent dans son parcours, tant professionnel que personnel : "J'ai le senti- ment d'avoir perdu trop d'années à faire des études. Je pensais qu'en n'ayant aucun problème judiciaire, en étant très actif dans la vie associative, voire dans la vie politique, qu'en suivant des études, qu'en restant dans le droit chemin en suivant les conseils de professeurs que j'avais, notamment un Iranien, qui me disait «si le Français il a 12, il faut que tu aies 14, s'il fait un pas en avant, il faut que tu en fasses deux pour être au même niveau ॥... C'est ce que j'ai essayé de faire au vu de mes résultats scolaires... [...] Le pire c'est que moi, pendant des années, $j$ 'ai poussé des petits jeunes en étant animateur dans différents quartiers, et j'étais là à leur dire qu'il faut travailler, aller à l'école, etc. Quand j'en voyais un dans mon quartier à rien foutre ou des conneries ou quoi que ce soit, franchement, je n'hésitais pas à mettre la tarte sur la tête, à discuter avec lui...Aujourd'hui, je ne me le permettrais plus. Premièrement parce que j'ai eu une remarque il y $a$ à peu près un an et demi sur " Et toi B., qu'est ce que tu deviens? Qu'est ce que tu fais ? Tu as étudié et qu'est ce que tu as ? " c'était heu.... Et deuxièmement, je ne peux pas, je ne peux plus parce que je sais que c'est les envoyer... C'est leur donner de faux espoirs, c'est honnêtement de faux espoirs" Cette situation montre à quel point la discrimination façonne le rapport au monde des personnes qui la subissent.

Face à cette souffrance sociale, la fonction d'écoute des permanences d'accueil du MRAP est primordiale. Elle permet non seule- 
ment la libération mais également la reconnaissance d'une parole qui a longtemps été perçue comme illégitime, non fondée, voire anecdotique. Au point que les victimes elles-mêmes peuvent apparaître surprises de se faire non seulement entendre, mais écouter : "Mais là je suis étonnée hein! vous êtes deux Françaises et vous m'écoutez! ". "Vraiment, merci beaucoup de m'avoir écouté ! Ça fait du bien! Parce qu'à la fin, personne ne nous croit ! ». Le déni qui entoure les questions de discriminations racistes oriente fréquemment le discours des victimes, et donne lieu à des stratégies discursives de persuasion de leur part. Face à l'accueillant, les plaignants peuvent être considérés comme étant « en représentation ", au sens goffmanien du terme : ils contrôlent l'impression qu'ils produisent sur l'interlocuteur en se mettant en avant sous un angle le plus positif possible (Goffman, 1963): "Je suis Français, je cotise, mes enfants sont Français, ma vie est ici, je respecte la loi, je suis citoyen ". «Mon casier judiciaire est vide, je n'ai jamais eu de problème avec la justice ». Dans leur argumentaire, il est aussi fréquent que les victimes anticipent une accusation de paranoïa ou d'exagération de la part de leurs interlocuteurs: "Je sais que ça peut paraître gigantesque comme histoire... ". "Je ne veux pas paraître paranoïaque, mais... ". "Au départ, je me disais que ce n'était pas vrai.Je ne voulais pas tomber dans la parano ". Toutes ces stratégies discursives ne suffisent pourtant pas toujours à « convaincre » l'accueillant.

Ici, on ne peut toutefois dissocier l'écoute de l'endroit dans laquelle elle se tient (une association " antiraciste ") ni des acteurs sociaux qui la mettent en œuvre (des militants). Comme je l'ai déjà mentionné, l'activité militante est avant tout une pratique sociale et politique sous-tendue par la volonté d'agir sur le réel. Si l'on peut, dans un souci heuristique, dissocier la vocation "d'écoute " et la vocation de " soutien ", dans la réalité, les deux se chevauchent. Pour le militant, l'écoute en tant que telle est toujours accompagnée par la volonté d'objectiver, ou du moins de mettre en lumière les faits proprement racistes. Or cette volonté peut être entravée par la structure même de l'accueil, qui ne met en jeu que la parole de la victime, ce qui implique un point de vue individuel, donc subjectif. La partialité des propos qu'ils recueillent est alors fréquemment mise en avant par les accueillants : "Il y a des versions qu'ils nous donnent, mais c'est les leurs ». "On est obligé de croire sur parole des gens qui nous cachent des choses. On n'a qu'une seule vision des choses. Or ils peuvent être responsables non pas de la discrimination, mais des actions qu'on peut avoir envers eux: ils ne parlent pas des fautes qu'ils ont pu connaître euxmêmes. On est alors amené à démêler des situations de gens qui ont un échec qu'ils octroient au racisme alors que c'est de leur responsabilité ॥. Cette remise en cause relève, de la part des militants, d'un 
refus de toute instrumentalisation du racisme. Ils y sont d'autant plus sensibles qu'ils se sentent manipulés dans une fonction qu'ils remplissent bénévolement pour la plupart, et qui leur demande un investissement personnel et affectif important dû à la difficulté que représente l'écoute de la souffrance d'autrui. L'aspect altruiste de leur activité ne semble pas pouvoir être totalement exempte d'un certain moralisme, du fait des convictions et des valeurs qui la sous-tendent et la motivent.

Pourtant, la délibération sur la véracité de la discrimination raciste que reflètent ces paroles est également révélatrice de la difficulté qu'il y a à saisir, à dire et à reconnaître le racisme pour les militants. Cette difficulté n'est d'ailleurs pas le seul fait de ces derniers, puisque les victimes, elles aussi, font état d'une interrogation sur le bien fondé de leurs propres doléances : «e ne sais pas quelle est la part du racisme dans ce qui m'est arrivé ». "Ce qui m'amène chez vous c'est un problème de relation, d'exclusion, de mépris, de disqualification, de discrimination [...]. Alors je ne sais pas quelle est la part de ce qu'on peut considérer comme exclusion raciale, et tout ce qui est de l'ordre de l'exclusion purement professionnelle ». "C'est vrai que les éléments sont minces. Les éléments de racisme pur, d'atteinte purement liée à l'origine. Il n'y a rien dans les propos ou les choses comme ça.De temps en temps on entend des choses, parce qu'on a beaucoup d'assistantes maternelles qui sont ma- ghrébines. Mais il n'y a pas... Je ne sais pas ॥. On peut toutefois se demander si, paradoxalement, la difficulté (voire, la réticence) à qualifier sans entrave le racisme n'est pas lié à la trop grande force d'accusation du terme. En effet, l'épithète " raciste ", lorsqu'elle est accolée à une personne ou à un comportement, s'apparente à une sentence, du fait de la réprobation morale collectivement partagée qui l'entoure. La puissance symbolique du terme peut alors faire hésiter quant à son utilisation.

C'est en grande partie cette immoralité du racisme qui pousse les militants à le dénoncer. $\mathrm{Ce}$ moralisme, qui imprègne une perception dominante de l'anti-racisme, s'accompagne la plupart du temps de la recherche d'une individualisation de la culpabilité, de façon à pouvoir personnifier, et donc circonscrire le « mal ». Or, la recherche systématique de cette dénonciation individuelle occulte la prise en compte d'une dimension plus structurelle du racisme et de la discrimination. Car, finalement, les catégories ethnistes et racistes imprègnent la structure même de la société française, et font partie intégrante des rapports sociaux. Elles sont non seulement disponibles, mais elles font sens et s'inscrivent dans un univers social qui rend possible leur usage de classification, donc de hiérarchisation, univers qu'elles influencent et qui, dans le même temps, contribue à les reproduire et à les légitimer. Le passage d'une prise en compte individuelle à une prise en compte 
plus collective du racisme et des discriminations suppose donc la contestation d'un ordre social inégalitaire et raciste ce qui s'avère plus difficile à instaurer que la dénonciation de comportements certes condamnables et odieux, mais qui ne sont que les résultats de ce premier.

\section{Notes}

${ }^{1}$ Souligné par nous.

${ }^{2}$ Par militants, j'entends dans ce texte les membres du MRAP qui s'investissent de façon régulière dans les activités et dans le cadre de cette association. La plupart le font bénévolement, mais certains sont salariés. Ici, je n'entrerai cependant pas plus dans les détails de ce qu'impliquent ces différences de statut.

3 Mon doctorat (Université de Grenoble-Stendhal) tout comme l'enquête que je mène auprès du MRAP étant, à ce jour, toujours en cours, les résultats exposés ici doivent être considérés comme intermédiaires et partiels.

${ }^{4}$ Rouen, Lille, Grenoble, St Etienne, Paris, Montpellier, Rennes et Toulouse.

${ }_{5}$ Tout au long de ce texte, je m'appuierai sur des citations soit de victimes, soit de militants du MRAP. $\mathrm{Ma}$ volonté étant de me concentrer sur ce couple "victime-militant », je ne donnerai pas d'indication autre que l'appartenance à l'une de ces deux catégories. Ce choix a également été influencé par la méthode utilisée pour collecter les données dont sont issus ces extraits d'entretiens - l'observation non participante - et qui a impliqué que je n'intervienne pas du tout dans l'interaction plaignant/écoutant : je ne dispose donc pas systématiquement des caractéristiques sociales telles que l'âge ou la catégorie socioprofessionnelle des personnes citées et ne peux donc y faire référence de manière pertinente.

${ }^{6}$ Il est très difficile de disposer de chiffres concernant le contentieux " discrimination ». Pour donner une idée de la faiblesse de ce contentieux, j'avancerai les statistiques fournies par la section du parquet de Paris qui traite le suivi des plaintes pour discrimination et racisme. En 2000, sur 289 plaintes déposées, 38 concernaient des cas de discrimination, et parmi eux, 24 ont été classées sans suite, quatre ont été jugées, les 10 restantes étant en attente de jugement.

\section{Bibliographie}

Bouamama, Saïd et Ahmed Benyachi, 2000. Les discriminations dans l'emploi et leurs impacts. L'exemple roubaisien. Voix de Nana, $92 \mathrm{p}$.

De Rudder, Véronique, Christian Poiret et François Vourc'h, 2000. L'inégalité raciste, L'universalité républicaine à l'épreuve. Paris, Presses universitaires de France, $201 \mathrm{p}$.

Essed, Philomena, 1991. Understanding Everyday Racism. An Interdisciplinary Theory. London Sage publication. Sage Series on race and ethnic relations. Vol. 2, 320 p.

Fassin, Didier, 2002. « L'invention française de la discrimination », Revue Française de Science Politique, no 4, vol. 52, août. 
Goffman, Erving, 1963. Stigmate. Les usages sociaux du handicap. Les éditions de minuit, Paris, $169 \mathrm{p}$. 